\title{
A Fuzzy Quality Function Deployment Approach for Differentiating Cloud Products
}

\author{
S. Emre Alptekin ${ }^{1}$, Gülfem Isiklar Alptekin ${ }^{2}$ \\ ${ }^{1}$ Department of Industrial Engineering, Galatasaray University, \\ Çırağan Cad. No. 3634357 Beşiktaş/İstanbul, TURKEY \\ E-mail: ealptekin@gsu.edu.tr \\ ${ }^{2}$ Department of Computer Engineering, Galatasaray University, \\ Çırağan Cad. No. 3634357 Beşiktaş/İstanbul, TURKEY \\ E-mail: gisiklar@gsu.edu.tr
}

Received 13 January 2017

Accepted 25 April 2018

\begin{abstract}
Cloud computing is defined as an on-demand large-scale distributed network to provide and realize computational resources. This flexibility expectation of cloud products forces cloud service providers (CSPs) to tailor their products to the needs of their customers. The framework in this paper proposes a fuzzy logic-based decision support tool for CSPs guiding them in differentiating their cloud offerings for different customer profiles. The tool aims to replicate human reasoning process by making use of fuzzy logic as a computational intelligence framework. The quality function deployment (QFD) approach in the tool systematically handle the product design process. The applicability of the proposed framework is shown using a real life cloud product design scenario with three customer profiles.
\end{abstract}

Keywords: Cloud computing; product design; fuzzy logic; quality function deployment, analytic network process; sustainability.

\section{Introduction}

The main thrust of cloud computing is to provide ondemand computing services with high reliability, scalability and availability in a distributed environment. ${ }^{1}$ Despite its several definitions, the most cited cloud computing definition is the one of National Institute of Standards and Technology: "Cloud computing is a model for enabling convenient on-demand network access to a shared pool of configurable computing resources (e.g., networks, servers, storage, applications, and services) that can be rapidly provisioned and released with minimal management effort or service provider interaction." It is seen as a new wave in the field of information technology. Refs. 2-4 differentiate cloud services and create three classes: SaaS (Software as a Service), PaaS (Platform as a Service) and IaaS (Infrastructure as a Service). Although in cloud computing, everything may be treated as a service (XaaS), in this research, we will concentrate on designing cloud services provided as IaaS. IaaS involves processing, storage, network and other fundamental computing resources that cloud customers utilize to deploy and run operating systems and software.

According to Ref. 5, public cloud services market will grow $17.2 \%$ in 2016 to total $\$ 208.6$ billion. This growth is expected to peak in 2017 to $18 \%$ and to plateau or slightly decrease through 2020 to a growth of $14.7 \%$. The highest grow is expected to come from IaaS. Amazon Web Services (AWS) has been the leader in the cloud infrastructure services market since 2006. In one of the recent studies, AWS and Microsoft are considered as leaders, Google is considered as visionary and IBM, VMware, etc. are considered as niche players. ${ }^{6}$ Ref. 7 states that AWS has the $31 \%$ of market share, followed 
by Microsoft with $11 \%$, IBM with 7\%, and Google with $5 \%$ in Q2 2016, respectively.

The potential benefits of cloud services can be achieved by adopting the right product. The growth in the number of cloud computing users has led different CSPs to offer a variety of cloud products. Hence, in literature, numerous research concentrate on the user's perspective for selecting the most suitable product in respect to their requirements. However, the challenge that the CSPs meet for designing/offering a variety of cloud products for a huge pool of customers is even harder. The design of any customized product must correspond to users' requirements to reach their expectations and these expectations does not necessarily converge. As a cloud product is exactly a customized product, its design process needs to follow the same steps. The success of AWS, that has been the global market leader for so long, may be attributed to their vast variety of offerings. On the other hand, having too many comparable options is mentally draining since each one must be weighed against each other. According to overchoice theory, as the number of choices increases and passes a certain point, people start to feel more pressure and get confused. ${ }^{8}$ This constitutes our motivation to propose an intelligent cloud service design process in order to come up with services matching needs of diverse customer profiles without overcomplicating the selection process.

Furthermore, the highly competitive cloud market together with the increasing pricing pressure necessitates a product of high quality with a cost conscious design. Also, as the intensity of cloud usage increases along with the need to save maximum possible amount of resources, the importance of sustainability increases. More and more CSPs have been caring for sustainability and especially for energy efficient computing initiatives, which complicates the matter further. In one of our recent works, we have proposed a decision support tool for ranking CSPs considering only customers' qualityrelated criteria. ${ }^{9}$ The proposed framework has taken into account a single customer profile and has used this input together with the CSPs' performance values to rank the cloud service offerings. In a succeeding work, we have proposed a decision support tool for producing cloud services considering three conflicting criteria; quality, cost and sustainability. ${ }^{10}$ The difference between the former and the latter studies is that the latter focuses specifically on the design process of CSP, while the former aims to support the selection decision of customer. However, the latter study still used a single customer profile and overlooked the ambiguous nature of the customers' judgments. In this paper, we intend to build a comprehensive decision support framework for CSPs in designing the most satisfactory product regarding different customer profiles considering quality alongside with cost and sustainability and also make use of fuzzy set theory to deal with subjective judgments of customers. ${ }^{11}$ Doing so, we exploit QFD approach, a common methodology, which intends to satisfy customers' needs $(\mathrm{CNs})$.

QFD methodology starts with the development of the house of quality (HOQ). HOQ uses customer feedbacks as input which are expressed as needs. It transforms this knowledge into product attributes, which represent technical attributes of a product. During the transformation process of $\mathrm{CNs}$ into the product technical requirements (PTRs), relationships between $\mathrm{CNs}$ and PTRs, and correlation among CNs and PTRs need to be resolved. This transformation enables obtaining the importance weights of PTRs. Thus, most important characteristics to address customers' concerns are identified. The gathering of this knowledge necessitates delicate handling of user feedback, usually expressed in user's own words in a natural language. In this paper, the vague nature of this knowledge has been overcome by using linguistic values represented by fuzzy numbers. It is believed that this will avoid oversimplification of a complex problem. Here, the weights are procured utilizing analytic network process (ANP). ${ }^{12}$ ANP is a generalization of Saaty's analytic hierarchy process (AHP), which is a universally adopted multi-criteria decision support tool. ${ }^{13}$ In most of the cases, the interaction and dependence of higher level elements on lower level elements complicate building a hierarchy for most of the real life decision problems. In this context, ANP and its supermatrix technique is introduced as an extension of AHP that can handle more complex decision structures $^{14,15}$. Although ANP framework has the ability to examine more elaborate interrelationships, it does not take into account the uncertainty associated with decision makers' judgments. Besides, the subjective judgments have strong influences in the ANP-based decisions. In decision making literature, deterministic/probabilistic sensitivity analysis, Bayes theorem, Dempster-Shafer theory, fuzzy set theory, and grey theory are among the commonly used approaches to deal with uncertainty. There are four classes of uncertainties: $i$. Vagueness, 
when information is naturally graded, ii. Imprecision, when the available information is not specific, iii. Ambiguity, when information leads to several possible interpretations, and $i v$. Inconsistency, when two or more information cannot be true at the same time. ${ }^{16}$ In our case, the decision makers' evaluations, generally, do not have sharp cutoff points, preferences are vague and have diversified meanings. Using precise numbers may oversimplify subjectivity. In order to overcome these shortcomings, fuzzy sets theory is used.

The main contribution of the recommended tool is that it enables CSPs to consider distinct and mostly contradicting issues simultaneously. Focusing only on the quality issues may satisfy customers, but it could hurt the profitability of the provider, if the cost of providing the service in long term is somehow neglected. Moreover, CSPs need to improve their chances of continuity in long term by responsively managing and maintaining their business resources. The proposed approach balances these three design goals (quality, cost and sustainability) for different customer segments (regular end-users, academic institutions, commercial institutions, etc.).

The paper is structured as follows. Section 2 outlines similar research in academic literature. The methodologies practiced in the framework are briefly presented in Section 3, while Section 4 gives step by step explanation of QFD-based decision support tool and its application. Section 5 displays the results of the case study and the concluding remarks with future works are offered in Section 6.

\section{Related Work}

In one of the recent studies on the cloud service selection problem, the authors have proposed a model by combining the feedback coming from cloud users and objective performance analysis from a trusted third party. ${ }^{17}$ In this work, the authors have applied a fuzzy simple additive weighting system. Ref. 18 has focused to select the preferable SaaS product in terms of functionality, architecture, usability, vendor reputation and cost. Their research is mainly based on subjective assessment, and they have made use of the AHP approach. Another research whose objective is to guide service consumers and providers in analyzing available Web services relies on fuzzy TOPSIS approach. ${ }^{19}$ The authors have ranked available alternative Web services with fuzzy opinions according to group preferences. Ref. 20 has designed a framework ("Cloudgenius") specifically for Web server migration to the cloud and defined a model and related factors for automating the decision making process. Their framework is based on AHP similar to the one in Ref. 14. The work that has more common traits with our study is the Ref. 21. In this work, the authors have first determined cloud key performance factors (KPIs). They have used AHP for assigning weights to features. Ref. 22 is another research that concentrate on selecting the suitable SaaS software package. Their ANP-based model considers both the intangible and tangible criteria by taking the interdependence and feedbacks into consideration.

These related works generally have the objective of selecting the most appropriate cloud product given the customer priorities. However, the proposed framework in this paper introduces an analytical approach for cloud service design. Ref. 21 has stated that they will extend their proposed quality model with non-quantifiable QoS attributes and deal with variations in attributes by applying fuzzy sets. In this paper, we used their attribute set. We then obtained a customer-oriented product design by additionally considering the dependencies among attributes using ANP, which was omitted in Ref. 21 due to the choice of AHP. Besides quality issues, we introduced the concept of cost conscious and sustainability conscious design. We wanted to show that a complete design should satisfy all stakeholders; not only the customers, but also the CSPs and the environmental agencies. As planned by Ref. 21, we made use of fuzzy sets in order to deal with the complexity and vagueness of the problem on hand.

In related literature, there are various research incorporating QFD and fuzzy ANP methodologies into different problem domains. ${ }^{23,24,25}$ The basic idea of the proposed framework in this paper is that it integrates customer feedback into cloud service design by considering distinct and contradicting goals simultaneously. Initial step for this procedure is to differentiate customers into profiles regarding their common expectations. The QFD methodology then identifies the interrelationships among customer feedback and the interrelationships among technical attributes. The technical attributes are CSPs' tools to modify the current product offering. In this manner, attributes that may be ignored (i.e. having little or no meaning to customer) are identified. Then, along with 
quality expectations, cost and sustainability are incorporated into the decision making process.

\section{The Methodologies}

\subsection{Quality function deployment (QFD)}

QFD methodology is a strategic knowledge management tool that brings together customer reactions and the product development process. Numerous information from various functional business units are brought together in QFD. The ultimate goal is to use these data to build a profitable and satisfactory product/service. QFD framework has a systematic approach to convert customer feedbacks to meaningful design-related knowledge. QFD methodology mainly stands on matrix operations. In each step of QFD, various information inputs are transformed to various outputs through matrices, where each step's output becomes the input of the following one. ${ }^{26}$ QFD starts with an initial matrix, called house of quality (Figure 1). The essential eight elements of HOQ can be outlined as:

(i) Customer needs (CNs) (WHATS). These are the customers' phrases where they describe their expectations from the service/product. They are usually called 'voice of the customer'.

(ii) Product technical requirements (PTRs) (HOWs). These are the measurable technical/design characteristics which help companies to respond to CNs. They are usually called 'voice of the company'.

(iii) Relative importance of the CNs. Simultaneously handling all the input coming from the customers is usually quite complicated. Eliminating insignificant $\mathrm{CNs}$ is chosen as a solution to this problem. Therefore, insignificant CNs needs to be eliminated.

(iv) Relationships between CNs and PTRs. These relationships constitute the core part of the HOQ. They define to what extent each PTR affects each $\mathrm{CN}$ resulting in importance values of $\mathrm{CNs}$ in terms of PTRs.

(v) Inner dependencies among the CNs. At this stage, the interactions among the $\mathrm{CNs}$ are found out. The predicted results can be utilized to measure how much and whether or not CNs supports each other.

(vi) Inner dependencies among the PTRs. Similar to the inner dependencies among CNs, the inner dependencies among PTRs are calculated and placed in the roof of HOQ. (vii) Competitive analysis. This step is crucial when both defining a marketing strategy and creating a sustainable competitive advantage. It incorporates competitor' performances into the decision process of CSPs when defining improvement directions.

(viii) Overall priorities and performance values of PTRs. The performance values of PTRs and additional goals are considered to form the final ranking of the PTRs.

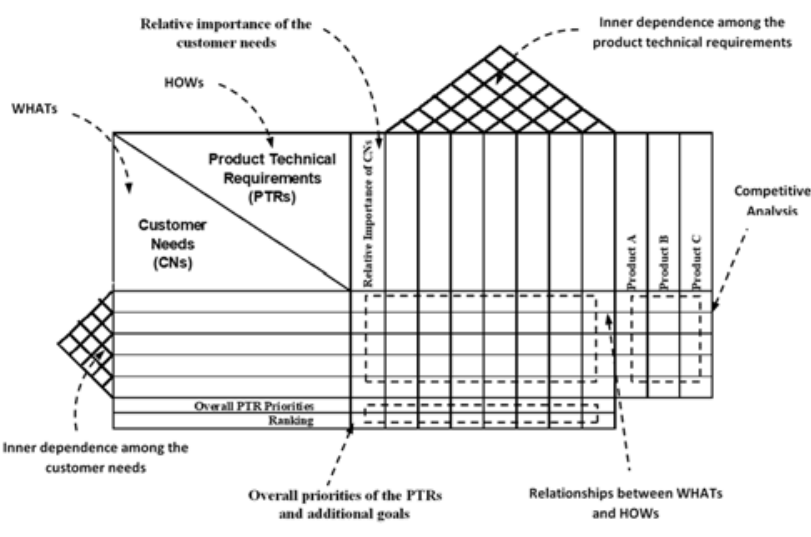

Fig. 1. House of quality

\subsection{Analytic Network Process}

ANP is accepted as an extension to the analytic hierarchy process. AHP separates a complex problem into several levels so that they constitutes a hierarchy. ${ }^{13}$ Each element in this hierarchy is assumed to be independent. Although AHP merges both qualitative and quantitative perspectives into a decision problem, it is not able to handle the interconnections and inner dependencies among decision factors at the same hierarchy level. ${ }^{27}$ For this purpose, ANP approach is developed, including networks instead of hierarchies (Figure 2). ${ }^{12}$ Hence, ANP can deal with the cases where there is interdependence among the alternatives and criteria.
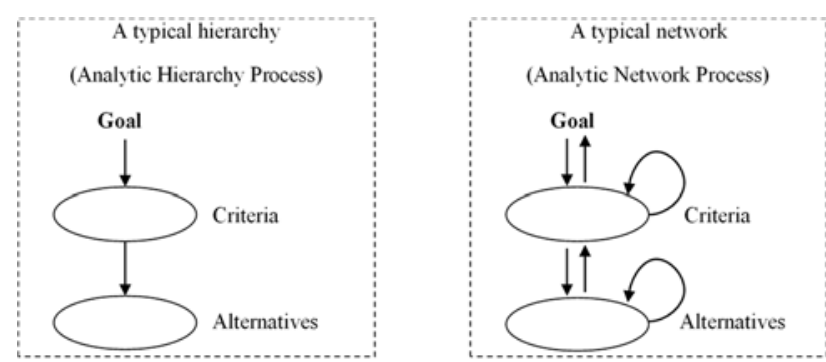

Fig. 2. Representation of AHP and ANP structures 
Customers' needs obtained as part of the QFD methodology are usually expressed in linguistic terms. Thus, vague and ambiguous data has to be translated. Fuzzy set theory as proposed by Ref. 28 defines the necessary elements to deal with the uncertainty due to imprecision and vagueness. Therefore, in this paper fuzzy ANP methodology is applied. Although the existence of many approaches in the literature that show how to apply fuzzy set theory to incorporate linguistic variables into calculations, due to simplicity of the calculations, we have used Chang's fuzzy extent analysis in this paper. ${ }^{29}$ Accordingly, an object set $X=$ $\left\{x_{1}, x_{2}, \cdots, x_{n}\right\}$ and a goal set $G=\left\{g_{1}, g_{2}, \cdots, g_{m}\right\}$ is defined. Extent analysis is performed taking each object and repeating the procedure for each goal resulting in $m$ fuzzy triangular values:

$M_{g_{i}}^{1}, M_{g_{i}}^{2}, \cdots, M_{g_{i}}^{m}, i=1,2, \cdots, n$

The linguistic scale and corresponding triangular fuzzy numbers are illustrated in Table 1 based on Saaty's scale. ${ }^{13}$ The parameter $\theta$ is taken as 1 .

Table 1. Linguistic scale and corresponding fuzzy numbers

\begin{tabular}{c|l}
\hline Fuzzy Number & Membership function \\
\hline$\tilde{1}$ & $(1,1,2)$ \\
$\tilde{x}$ & $(x-1, x, x+1)$ for $x=2,3,4,5,6,7,8$ \\
$\tilde{9}$ & $(8,9,9)$ \\
\hline
\end{tabular}

Stepwise representation of extent analysis is given as follows ${ }^{30}$ :

Step 1 . The value of fuzzy synthetic extent with respect to the $i^{\text {th }}$ object is calculated as,

$S_{i}=\sum_{j=1}^{m} M_{g_{i}}^{j} \otimes\left[\sum_{i=1}^{n} \sum_{j=1}^{m} M_{g_{i}}^{j}\right]^{-1}$

The details of the necessary fuzzy operations are explained in Ref. 29 and Ref. 30.

Step 2. The degree of possibility of $M_{2}\left(l_{2}, m_{2}, u_{2}\right) \geq$ $M_{1}\left(l_{1}, m_{1}, u_{1}\right)$ is defined as:

$V\left(M_{2} \geq M_{1}\right)=\sup _{y \geq x}\left[\min \left(\mu_{M_{1}}(x), \mu_{M_{2}}(y)\right)\right]$

and can be expressed as demonstrated by Ref. 30,

$V\left(M_{2} \geq M_{1}\right)=\operatorname{hgt}\left(M_{1} \cap M_{2}\right)=\mu_{M_{2}(d)}$

$\mu_{M_{2}(d)}=\left\{\begin{array}{c}1 \\ 0 \\ \frac{l_{1}-u_{2}}{\left(m_{2}-u_{2}\right)\left(m_{1}-l_{1}\right)}\end{array}\right.$ where $d$ is defined as the ordinate of the highest intersection point $D$ between the two triangular fuzzy numbers $\mu_{M_{1}}$ and $\mu_{M_{2}}$. The methodology requires the calculation of both $V\left(M_{1} \geq M_{2}\right)$ and $V\left(M_{2} \geq M_{1}\right)$.

Step 3 . The degree possibility for a convex fuzzy number to be greater than $k$ convex fuzzy numbers is defined by

$V\left(M \geq M_{1}, M_{2}, \cdots M_{k}\right)=V\left[\left(M \geq M_{1}\right)\right.$ and $(M \geq$ $\left.M_{2}\right) \cdots$ and $\left.\left(M \geq M_{k}\right)\right]$

$V\left(M \geq M_{1}, M_{2}, \cdots M_{k}\right)=\operatorname{minV}\left(\left(M \geq M_{i}\right)\right), i=$

$1,2,3 \cdots k$

If $d^{\prime\left(A_{i}\right)}=\operatorname{minV}\left(S_{i} \geq S_{k}\right), \quad$ then for $k=$ $1,2,3, \cdots, n ; k \neq i$, the corresponding weight vector can be calculated as follows:

$W^{\prime}=\left(d^{\prime}\left(A_{1}\right), d^{\prime}\left(A_{2}\right), \cdots, d^{\prime}\left(A_{n}\right)\right)^{T}$

Step 4. The last step calculates the normalized weight vectors:

$W=\left(d\left(A_{1}\right), d\left(A_{2}\right), \cdots, d\left(A_{n}\right)\right)^{T}$

where $W_{i}$ is a nonfuzzy number. ${ }^{29}$

\section{Research Framework}

In the proposed decision support framework, CSPs need to incorporate customer perspective in terms of quality expectations along with their own perspective in terms of sustainability and cost. The aim is balancing design preferences in a simple and systematic way. The research methodology is based on the model in Ref. 31. In that research, the authors use ANP with the QFD in order to recommend improvement goals for PTRs. Ref. 31's case study considers improving the design of a pencil. They incorporate customer feedback into the decision and design process. Then, they make use of a goal programming approach that takes into account all cost, extendibility and manufacturability dimensions. They build only one HOQ matrix to obtain a customer-oriented design, whereas in this paper we formed three HOQ matrices, one for customers' and the remaining two for CSPs' satisfaction, respectively. We then combine their results in order to obtain the most convenient cloud product both from customers and CSPs' perspectives. In other words, three HOQ matrices are used, one for the quality, one for the cost and another one for the sustainability goal.

Similar to Ref. 31, ANP is applied to prioritize design criteria. The strength of dependencies among the criteria 
are evaluated using pairwise comparisons. The influences of criteria on other ones are represented via a matrix, called a supermatrix. It is raised to powers until it converges to a limiting matrix with the overall priorities of the elements. ${ }^{32}$ This research uses the following supermatrix representation ${ }^{31}$ :

$$
\mathbf{W}=\begin{array}{ccc}
\mathrm{G} & \mathrm{C} & \mathrm{A} \\
\operatorname{Goal}(\mathrm{G}) \\
\text { Criteria }(\mathrm{C}) \\
\text { Alternatives (A) }
\end{array}\left(\begin{array}{ccc}
0 & 0 & 0 \\
\mathbf{w}_{1} & \mathbf{W}_{3} & 0 \\
0 & \mathbf{W}_{2} & \mathbf{W}_{4}
\end{array}\right)
$$

where

$\mathbf{w}_{1}$ : A vector representing the impact of the goal,

$\mathbf{W}_{2}$ : A matrix denoting the impact of the $\mathrm{CNs}$ on each of the PTRs,

$\mathbf{W}_{3}$ and $\mathbf{W}_{4}$ : The matrices representing inner dependencies of the CNs and PTRs, respectively.

We used fuzzy extent analysis methodology to convert fuzzy numbers into corresponding crisp weights and placed them in respective matrices.

The supermatrix representation given in (1) defines a network with two clusters (criteria and alternatives) besides the goal. Instead of raising the supermatrix to limiting powers, a matrix manipulation approach $\mathrm{h}^{33} \mathrm{can}$ be employed to obtain overall priorities of the elements. The approach suggests that, by multiplying $\mathbf{W}_{\mathbf{3}}$ with $\mathbf{w}_{\mathbf{1}}$, the vector of interdependent priorities of the $\mathrm{CNs}\left(\mathbf{w}_{\mathbf{C}}\right)$ and by multiplying $\mathbf{W}_{\mathbf{4}}$ by $\mathbf{W}_{\mathbf{2}}$, the matrix of interdependent priorities of the PTRs $\left(\mathbf{W}_{\mathbf{A}}\right)$ can be obtained. Overall priorities of the elements ( $\left.\mathbf{W}_{\mathbf{A N P}}\right)$ is the result of the multiplication of $\mathbf{W}_{\mathbf{A}}$ with wc. These weights correspond to the importance ratings for PTRs. Since this research combines three different goals, the same calculations are repeated three times with different aims. At the end, we obtained three sets of weights representing quality, cost and sustainability views. The preference of the CSP towards these views is calculated using pairwise comparisons. Finally, the overall weight vector of PTRs is computed using simple additive weighting approach. Figure 3 depicts the main steps and their precedence.

Step 1. The proposed design framework starts by defining the customer needs that represent the perceptions of customers regarding the product/service and are expressed using customer's own phrases. Then, the product technical requirements that represent the resources used to fulfill the CNs are defined.
Step 2. As customer needs are usually too diverse to deal with simultaneously, they have to be rated in order to determine the most important ones. These ratings will reflect the order of preference. Herein, as suggested by the ANP methodology, pairwise comparisons are implemented. For obtaining crisp weights we used fuzzy extent analysis. The result is the w1 vector. We differentiated cloud customers into three different profiles. Therefore, at this stage different $\mathbf{w}_{\mathbf{1}}$ vectors representing different customer profiles are calculated. Since there are three separate goals (quality, cost and sustainability) in our decision framework, the $\mathbf{w}_{\mathbf{1}}$ vector is calculated three times, each time with the respective goal.

Step 3. This step involves the identification of the degrees of relative importance of PTRs with respect to each $\mathrm{CN}$. In other words, whether or not and how much a PTR affects a $\mathrm{CN}$ is determined. Doing so, we assume that there is not any dependence among PTRs. The result of this step is given as $\mathbf{W}_{2}$. Similar to the calculations for $\mathbf{w}_{1}, \mathbf{W}_{\mathbf{2}}$ is calculated three times in order to reflect the effect of the different goals.

Step 4. In real life scenarios, the interactions are too complex to assume independence among the CNs. ANP is applied as the means to determine the interactions among CNs. Similarly; pairwise comparisons are used to obtain $\mathbf{W}_{\mathbf{3}}$.

Step 5. Similar to CNs, PTRs may affect each other. Therefore, the inner dependencies among them need to be determined. The results obtained using ANP is represented as $\mathbf{W}_{\mathbf{4}}$.

Step 6. At this stage, CNs need to be transformed into measurable PTRs. For this transformation, the interdependent priorities of $\mathrm{CNs}\left(\mathbf{w}_{\mathbf{C}}\right)$ and the interdependent priorities of PTRs $\left(\mathbf{W}_{\mathbf{A}}\right)$ are calculated. $\mathbf{W}_{\mathbf{A}}$ and $\mathbf{w c}_{\mathbf{C}}$ are then combined to obtain the overall priorities of PTRs for each respective goal.

Step 7. The proposed product design framework tries to balance three distinctive goals (quality, cost, and sustainability). Therefore, the CSP's preference towards these goals have to be determined. Herein, pairwise comparisons are once again utilized. The resulting relative importance weights are combined with overall priorities of PTRs for each goal with simple additive weighting approach. Building the hierarchy structure of cloud service with CNs and PTRs is a one-time effort and remains the same until there is a necessity for changing the set of CNs and PTRs. 


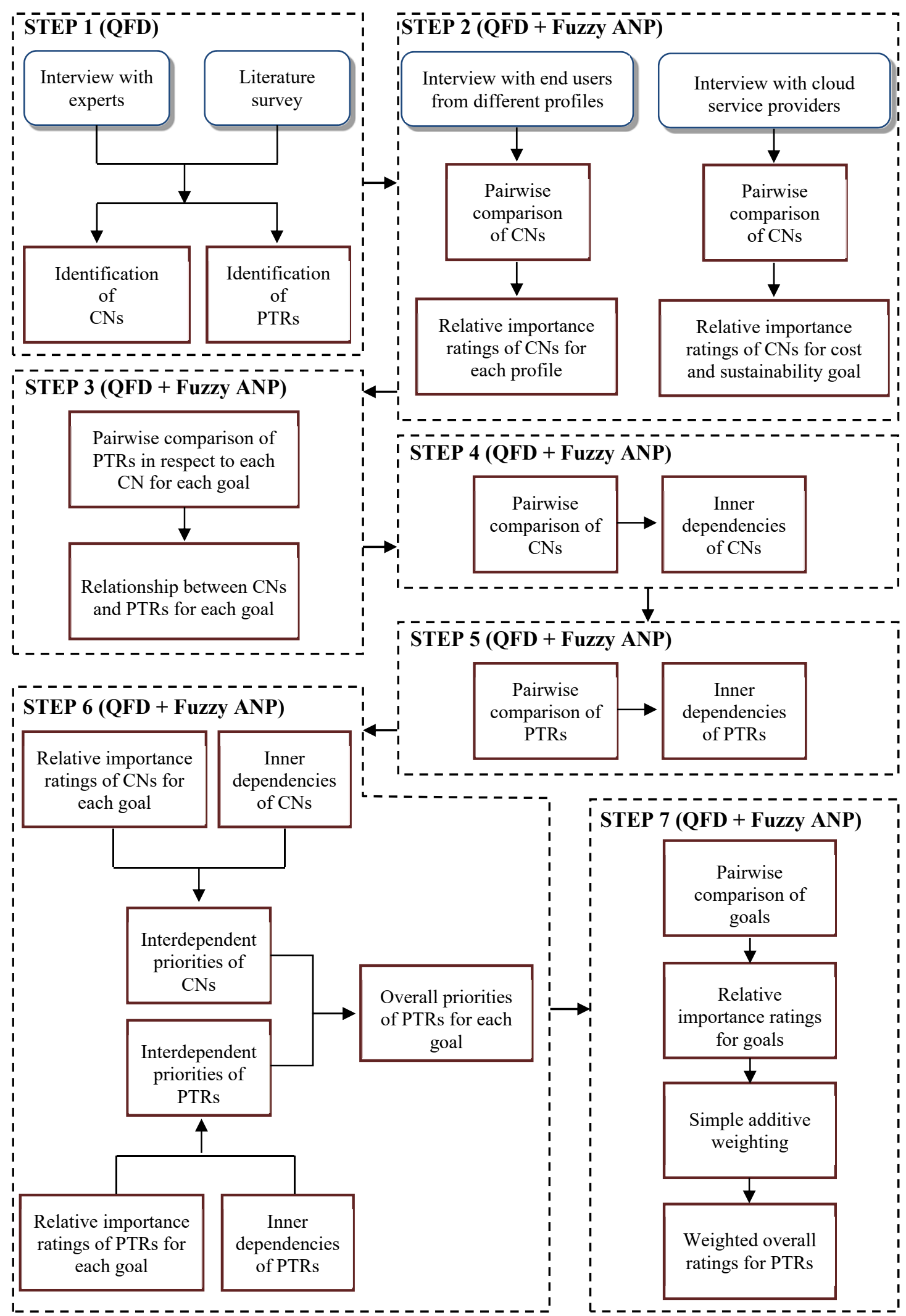

Fig. 3. Cloud product design framework 
The most time consuming step is the pairwise comparisons. The Miller's Law states that the number of objects that an average human can handle in short term memory is $7 \pm 2 .{ }^{34}$ Hence, when making pairwise comparisons, at most 9 comparisons are made. Fuzzy logic facilitates decision makers' work when making pairwise comparisons, since it resembles to human reasoning.

\section{Empirical Analysis}

In our demonstrative example, we aim at showing how the technical specifications of cloud products should be attuned according to different customer profiles. The data used in the example is based on the work of Ref. 21. They intended to select the best CSP among Amazon EC2, Windows Azure and Rackspace, considering only their quality aspects. Our previous study that used only a single profile made use of the same data set as well. ${ }^{10}$

Step 1. The CNs are specified using the first level QoS attributes defined in Ref. 21, since they established a comprehensive list. We utilize their attributes and classify quality-related attributes as $\mathrm{CNs}$ and performance related attributes as PTRs (Table 2). ${ }^{10}$ Their explanations are given in more detail in Ref. 21.

Table 2. List of customer needs and product technical requirements

\begin{tabular}{|c|c|}
\hline Customer Needs (CNs) & \begin{tabular}{|l} 
Product Technical \\
Requirements (PTRs)
\end{tabular} \\
\hline CN1: Accountability & $\begin{array}{l}\text { PTR1: Accountability } \\
\text { performance }\end{array}$ \\
\hline CN2: Capacity & PTR2: CPU capacity \\
\hline CN3: Elasticity & PTR3: Memory capacity \\
\hline CN4: Availability & PTR4: Disk capacity \\
\hline CN5: Service stability & $\begin{array}{l}\text { PTR5: Mean time taken to } \\
\text { expand/contract serv. capacity }\end{array}$ \\
\hline CN6: Serviceability & PTR6: Availability \\
\hline CN7: On-going cost & PTR7: Upload time \\
\hline CN8: Serv. response time & PTR8: CPU stability \\
\hline \multirow[t]{10}{*}{ CN9: Security } & PTR9: Memory stability \\
\hline & PTR10: Free support \\
\hline & PTR11: Type of support \\
\hline & PTR12: Virtual machine cost \\
\hline & PTR13: Inbound data cost \\
\hline & PTR14: Outbound data cost \\
\hline & PTR15: Storage cost \\
\hline & $\begin{array}{l}\text { PTR16: Service response time } \\
\text { range }\end{array}$ \\
\hline & $\begin{array}{l}\text { PTR17: Service response time } \\
\text { average value }\end{array}$ \\
\hline & PTR18: Security performance \\
\hline
\end{tabular}

Step 2. The relative importance of the CNs for the quality goal are determined by asking the following question: 'Which $\mathrm{CN}$ should be emphasized more in establishing the most satisfactory cloud product?'. The challenge at this stage is that usually CSPs have to deal with the expectations of extremely different customer profiles (for example, a firm of 900 employees vs. a startup business of two buddies). As differentiated customer requirements, we define three customer profiles (Table 3). The first one is the profile that Ref. 21 used in their case study. They randomly determined weights of customer needs. The second profile represents a scientific community. The importance weights for this profile are identified based on an interview with the head of the IT department of our university. The third profile represents a medium-sized established commercial organization, whose importance weights are calculated by taking the average of the results obtained from two CIOs of two medium-sized software development firms. For this demonstrative case study, the points of views of two CIOs are found sufficient; but a totally independent study may be done to determine and evaluate these criteria as a future work.

Table 3. Three different customer profiles

\begin{tabular}{c||l}
\hline \hline Customer Profiles & Description \\
\hline Profile 1 & $\begin{array}{l}\text { Ref. } 21 ; \text { weights of CNs are determined } \\
\text { randomly. }\end{array}$ \\
\hline Profile 2 & Scientific community (i.e. university) \\
\hline Profile 3 & $\begin{array}{l}\text { Commercial organization (i.e. medium- } \\
\text { sized firm) }\end{array}$ \\
\hline \hline
\end{tabular}

The resulting weights after applying Chang's fuzzy extent analysis for different profiles are illustrated in Figure 4. We can observe differences among weights, especially for CN1, CN2, CN3, CN8 and CN9. For instance, the weight of capacity (CN2) is $6 \%$ for profile $1,29.5 \%$ for profile 2 and is considered ignorable $(0 \%)$ for profile 3. This is an indicator of how different customer expectations could be.

The relative importance of the $\mathrm{CNs}$ for the cost and sustainability goals are determined by asking the same questions. However, as these goals represent CSPs' perspectives, we get in touch with a CTO from a CSP and let him make the pairwise comparisons. The resulting weights are given in Table 4 and Table 5. The results reveal that the three most important $\mathrm{CNs}$ affecting the cost of the cloud product are 'on-going cost', 'security' and 'capacity'. The three most important CNs affecting 
the cloud product in terms of sustainability are 'elasticity', 'on-going cost' and 'availability'.

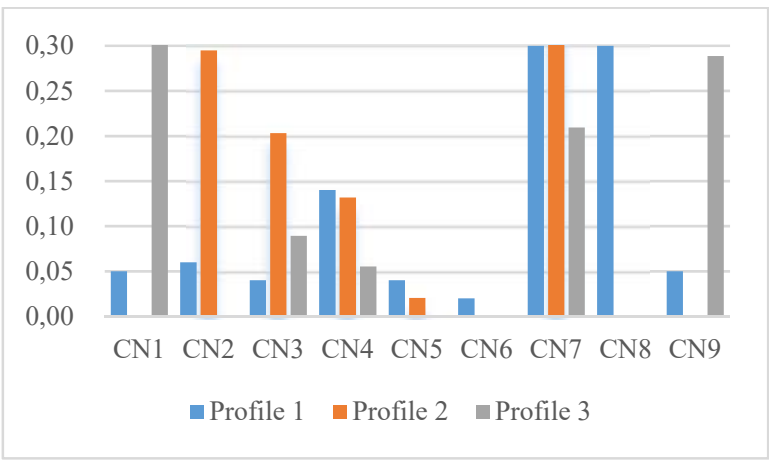

Fig. 4. Relative importance weights of CNs of different customer profiles for the quality goal

Table 4. Relative importance weights of CNs for the cost goal $\left.\begin{array}{l|lllllllll}\hline \mathbf{w}_{1} & (0.000 & 0.193 & 0.000 & 0.033 & 0.000 & 0.000 & 0.361 & 0.095 & 0.318\end{array}\right)^{T}$

Table 5. Relative importance weights of CNs for the sustainability goal

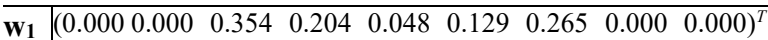

Step 3. Assuming that PTRs are independent, they are compared with respect to each $\mathrm{CN}$ considering different goals in mind. One example of questions is: 'What is the relative importance of CPU stability when compared to upload time on controlling service stability?'. Table 6 summarizes the relative importance of PTRs for all the $\mathrm{CNs}$ for the quality goal in mind $\left(\mathbf{W}_{\mathbf{2}}\right)$. The same calculations are done for the remaining goals (cost and sustainability).

Step 4. This step comprises the identification of the inner dependencies among the CNs. A possible question at this stage is: 'Given the $\mathrm{CN}$, availability, which $\mathrm{CN}$ contributes availability more, and how much more?' yielding to the inner dependence matrix given in Table 7 . We computed the normalized crisp weights using fuzzy extent analysis. The resulting column eigenvectors are summarized in Table $8\left(\mathbf{W}_{3}\right)$.

Step 5. In this step, we determine the inner dependencies among the PTRs similar to Step 4 and obtain $\mathbf{W}_{4}$ (Table 9).

Step 6. The overall priorities of the PTRs ( $\left.\mathbf{W}_{\text {ANP }}\right)$ are calculated using matrix manipulations as explained in Section 4. The results reveal the diversified nature of potential customers and its effect on the product requirements (Figure 5).

Table 6. Column eigenvectors with respect to each $\mathrm{CN}$ for the quality goal

\begin{tabular}{l|ccccccccc}
\hline $\mathbf{W}_{2}$ & CN1 & CN2 & CN3 & CN4 & CN5 & CN6 & CN7 & CN8 & CN9 \\
\hline PTR1 & 1.000 & 0 & 0 & 0 & 0 & 0 & 0 & 0 & 0 \\
PTR2 & 0 & 0.449 & 0 & 0 & 0 & 0 & 0 & 0 & 0 \\
PTR3 & 0 & 0.351 & 0 & 0 & 0 & 0 & 0 & 0 & 0 \\
PTR4 & 0 & 0.200 & 0 & 0 & 0 & 0 & 0 & 0 & 0 \\
PTR5 & 0 & 0 & 1.000 & 0 & 0 & 0 & 0 & 0 & 0 \\
PTR6 & 0 & 0 & 0 & 1.000 & 0 & 0 & 0 & 0 & 0 \\
PTR7 & 0 & 0 & 0 & 0 & 0.365 & 0 & 0 & 0 & 0 \\
PTR8 & 0 & 0 & 0 & 0 & 0.403 & 0 & 0 & 0 & 0 \\
PTR9 & 0 & 0 & 0 & 0 & 0.232 & 0 & 0 & 0 & 0 \\
PTR10 & 0 & 0 & 0 & 0 & 0 & 1.000 & 0 & 0 & 0 \\
PTR11 & 0 & 0 & 0 & 0 & 0 & 0 & 0 & 0 & 0 \\
PTR12 & 0 & 0 & 0 & 0 & 0 & 0 & 0.878 & 0 & 0 \\
PTR13 & 0 & 0 & 0 & 0 & 0 & 0 & 0 & 0 & 0 \\
PTR14 & 0 & 0 & 0 & 0 & 0 & 0 & 0 & 0 & 0 \\
PTR15 & 0 & 0 & 0 & 0 & 0 & 0 & 0.122 & 0 & 0 \\
PTR16 & 0 & 0 & 0 & 0 & 0 & 0 & 0 & 0.500 & 0 \\
PTR17 & 0 & 0 & 0 & 0 & 0 & 0 & 0 & 0.500 & 0 \\
PTR18 & 0 & 0 & 0 & 0 & 0 & 0 & 0 & 0 & 1.000 \\
\hline
\end{tabular}

Table 7. The inner dependence matrix for 'availability' $\mathrm{CN}$

\begin{tabular}{l|cccc}
\hline Availability & Availability & Serviceability & $\begin{array}{c}\text { Service } \\
\text { response time }\end{array}$ & Security \\
\hline Availability & 1 & $\tilde{2}$ & $\tilde{3}$ & $\tilde{3}$ \\
Serviceability & $\tilde{2}^{-1}$ & 1 & $\tilde{2}$ & $\tilde{3}$ \\
Service & $\tilde{3}^{-1}$ & $\tilde{2}^{-1}$ & 1 & $\tilde{1}$ \\
response time & $\tilde{3}^{-1}$ & $\tilde{3}^{-1}$ & $\tilde{1}^{-1}$ & 1 \\
Security & & & & \\
\hline
\end{tabular}

Table 8. The inner dependence matrix of CNs

\begin{tabular}{l|ccccccccc}
\hline $\mathbf{W}_{3}$ & CN1 & CN2 & CN3 & CN4 & CN5 & CN6 & CN7 & CN8 & CN9 \\
\hline CN1 & 1.000 & 0 & 0 & 0 & 0 & 0 & 0 & 0 & 0 \\
CN2 & 0 & 0.946 & 0 & 0 & 0 & 0 & 0 & 0 & 0 \\
CN3 & 0 & 0 & 0.678 & 0 & 0 & 0 & 0 & 0 & 0 \\
CN4 & 0 & 0 & 0 & 0.499 & 0 & 0 & 0 & 0 & 0 \\
CN5 & 0 & 0 & 0 & 0 & 0.616 & 0 & 0 & 0.384 & 0 \\
CN6 & 0 & 0 & 0 & 0.383 & 0.384 & 0.692 & 0 & 0. & 0 \\
CN7 & 0 & 0.054 & 0.322 & 0 & 0 & 0.308 & 1.000 & 0 & 0 \\
CN8 & 0 & 0 & 0 & 0.118 & 0 & 0 & 0 & 0.616 & 0 \\
CN9 & 0 & 0 & 0 & 0 & 0 & 0 & 0 & 0 & 1.000 \\
\hline
\end{tabular}

The main objective of our proposed framework is to guide CSPs in designing their cloud offerings. Thus, the overall ratings of PTRs serve for this purpose. They point out the specifications of the product on which the CSPs have to concentrate their efforts in order to satisfy the needs of their customers. For instance, for commercial organizations (Profile 3) 'accountability' (PTR1), and 'security performance' (PTR18) seem to be considerably more important compared to Profile 1 and Profile 2. On the other hand, for a scientific community, ' $V M$ cost' 
Table 9. The inner dependence matrix of PTRs

\begin{tabular}{|l|c|c|c|c|c|c|c|c|c|c|c|c|c|c|c|c|c|c|}
\hline W4 $_{4}$ & PTR1 & PTR2 & PTR3 & PTR4 & PTR5 & PTR6 & PTR7 & PTR8 & PTR9 & PTR10 & PTR11 & PTR12 & PTR13 & PTR14 & PTR15 & PTR16 PTR17 & PTR18 \\
\hline Accountability (PTR1) & 1.000 & 0 & 0 & 0 & 0 & 0 & 0 & 0 & 0 & 0 & 0.140 & 0 & 0 & 0 & 0 & 0 & 0 & 0 \\
\hline CPU capacity (PTR2) & 0 & 0.491 & 0 & 0 & 0.308 & 0 & 0 & 0.077 & 0 & 0 & 0 & 0.345 & 0 & 0 & 0 & 0 & 0 & 0 \\
\hline Memory capacity (PTR3) & 0 & 0 & 0.673 & 0 & 0 & 0 & 0 & 0 & 0.177 & 0 & 0 & 0.152 & 0 & 0 & 0 & 0 & 0 & 0 \\
\hline Disk (PTR4) & 0 & 0 & 0 & 0.399 & 0 & 0 & 0 & 0 & 0 & 0 & 0 & 0 & 0 & 0 & 0.366 & 0 & 0 & 0 \\
\hline $\begin{array}{l}\text { Mean time taken to expand or contract the service } \\
\text { capacity (PTR5) }\end{array}$ & 0 & 0 & 0 & 0 & 0.692 & 0 & 0 & 0 & 0 & 0 & 0 & 0 & 0 & 0 & 0 & 0 & 0 & 0 \\
\hline Availability (PTR6) & 0 & 0 & 0 & 0 & 0 & 0.946 & 0 & 0 & 0 & 0 & 0 & 0 & 0 & 0 & 0 & 0 & 0 & 0 \\
\hline Upload time (PTR7) & 0 & 0 & 0 & 0 & 0 & 0 & 1.000 & 0 & 0 & 0 & 0 & 0 & 0 & 0 & 0 & 0 & 0 & 0 \\
\hline CPU stability (PTR8) & 0 & 0 & 0 & 0 & 0 & 0.054 & 0 & 0.612 & 0 & 0 & 0 & 0 & 0 & 0 & 0 & 0.149 & 0.149 & 0 \\
\hline Memory stability (PTR9) & 0 & 0 & 0 & 0 & 0 & 0 & 0 & 0 & 0.483 & 0 & 0 & 0 & 0 & 0 & 0 & 0.113 & 0.113 & 0 \\
\hline Free support (PTR10) & 0 & 0 & 0 & 0 & 0 & 0 & 0 & 0 & 0 & 0.816 & 0 & 0 & 0 & 0 & 0 & 0 & 0 & 0 \\
\hline Type of support (PTR11) & 0 & 0 & 0 & 0 & 0 & 0 & 0 & 0 & 0 & 0 & 0.486 & 0 & 0 & 0 & 0 & 0 & 0 & 0 \\
\hline VM cost (PTR12) & 0 & 0.330 & 0.327 & 0.202 & 0 & 0 & 0 & 0.311 & 0.340 & 0 & 0.374 & 0.503 & 0 & 0 & 0 & 0 & 0 & 0 \\
\hline Inbound data cost (PTR13) & 0 & 0 & 0 & 0 & 0 & 0 & 0 & 0 & 0 & 0 & 0 & 0 & 1.000 & 0 & 0 & 0 & 0 & 0 \\
\hline Outbound data cost (PTR14) & 0 & 0 & 0 & & 0 & 0 & 0 & 0 & 0 & 0 & 0 & 0 & 0 & 1.000 & 0 & 0 & 0 & 0 \\
\hline Storage cost (PTR15) & 0 & 0 & 0 & 0.399 & 0 & 0 & 0 & 0 & 0 & 0 & 0 & 0 & 0 & 0 & 0.634 & 0 & 0 & 0 \\
\hline Service R. T. range (PTR16) & 0 & 0.128 & 0 & 0 & 0 & 0 & 0 & 0 & 0 & 0 & 0 & 0 & 0 & 0 & 0 & 0.304 & 0.304 & 0 \\
\hline Service R.T. average (PTR17) & 0 & 0.051 & 0 & 0 & 0 & 0 & 0 & 0 & 0 & 0.184 & 0 & 0 & 0 & 0 & 0 & 0.434 & 0.434 & 0 \\
\hline Security performance (PTR18) & 0 & 0 & 0 & 0 & 0 & 0 & 0 & 0 & 0 & 0 & 0 & 0 & 0 & 0 & 0 & 0 & 0 & 1.000 \\
\hline
\end{tabular}

(PTR12) and 'CPU capacity' (PTR2) are more decisive attributes. Therefore, CSPs have to attune their offerings accordingly.

The overall priorities of PTRs for cost and sustainability goals are calculated using the same matrix manipulations. The resulting weights are given in Figure 6. The first row represents the importance weights of product technical requirements when just quality goal is considered (profile 1), while the second and third rows represent the importance weights when the cost and sustainability goals are considered, respectively. The analysis results point out that the most important costoriented cloud service requirements are 'security performance', 'VM cost' and 'CPU capacity' and most important sustainability-oriented cloud service requirements are 'time', 'VM cost' and 'CPU capacity'.

Step 7. The CSP's attitude towards quality, cost and sustainability is determined using pairwise comparisons by the same CTO consulted for the previous calculations. The resulting relative importance ratings for each goal are summarized in Table 10. According to the ratings, quality attribute is considered more important than cost and sustainability with a weight of $43.9 \%$. For another decision maker with different priorities, cost or sustainability could become more important.
Table 10. Relative importance ratings for quality, cost and sustainability goals

\begin{tabular}{l|c}
\hline Goals & Relative importance ratings \\
\hline Quality & 0.439 \\
Cost & 0.329 \\
Sustainability & 0.233 \\
\hline
\end{tabular}

The overall ratings for PTRs are calculated using simple additive weighting by combining overall ratings of PTRs for each goal. Since there are three different profiles for the quality goal, for the sake of simplicity, only the first profile is depicted. In a real life scenario, CSPs could attach different weights for different customer profiles and may use simple additive weighting to obtain an overall rating for the quality goal or simply provide different service offerings for each customer profile. The last row in Figure 6 summarizes the unification of different views with the weighted overall ratings of PTRs for the first customer profile. The results reveal that overall the most meaningful cloud service attribute is ' $V M$ cost', followed by, 'security performance' and ' $C P U$ capacity' for a customer with the first profile and CSPs own cost and sustainability preferences. 


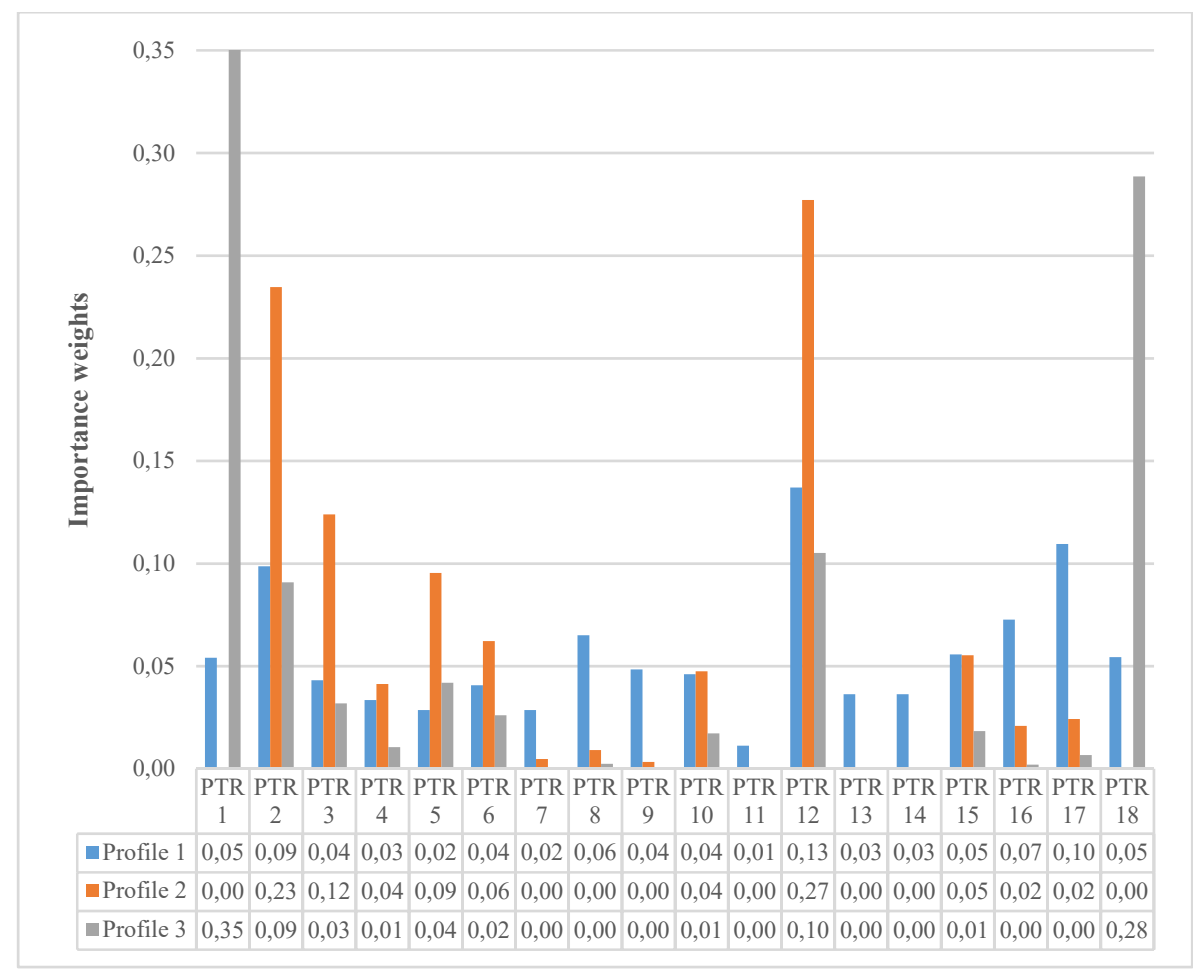

Fig 5. Overall priorities of PTRs for the quality goal with different profiles

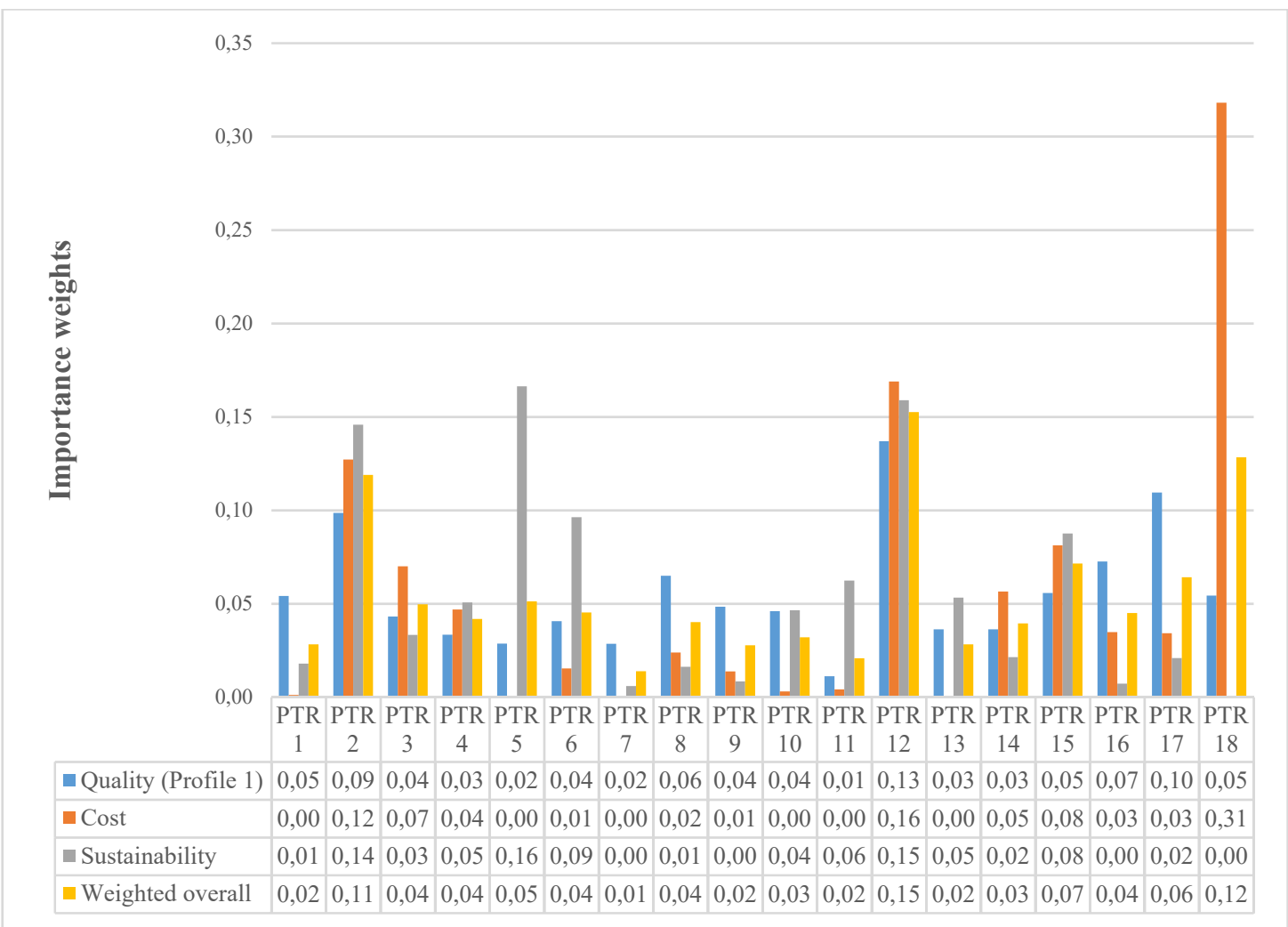

Fig 6. Overall priorities of PTRs for different goals 


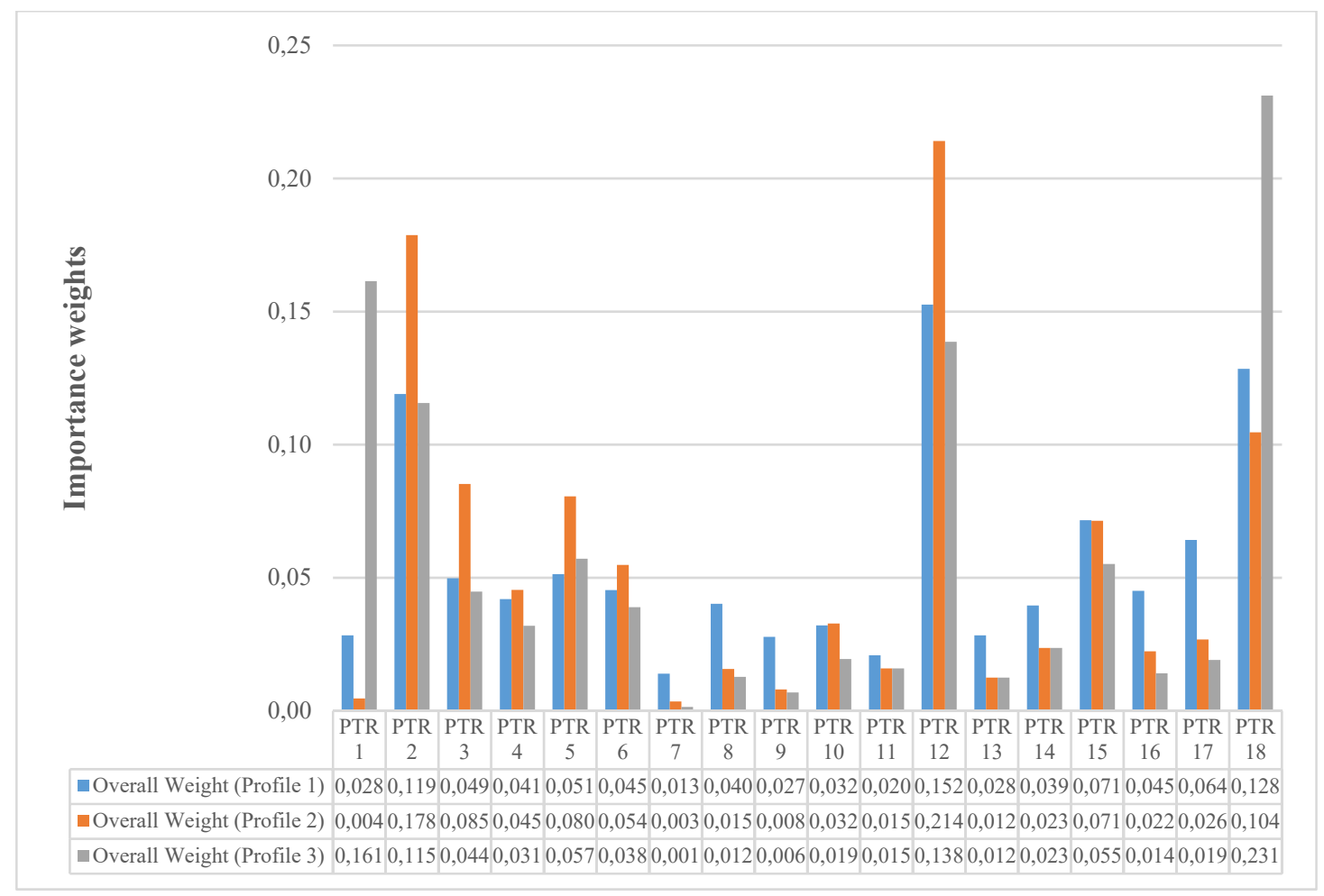

Fig. 7. Overall weighted priorities of PTRs for different profiles

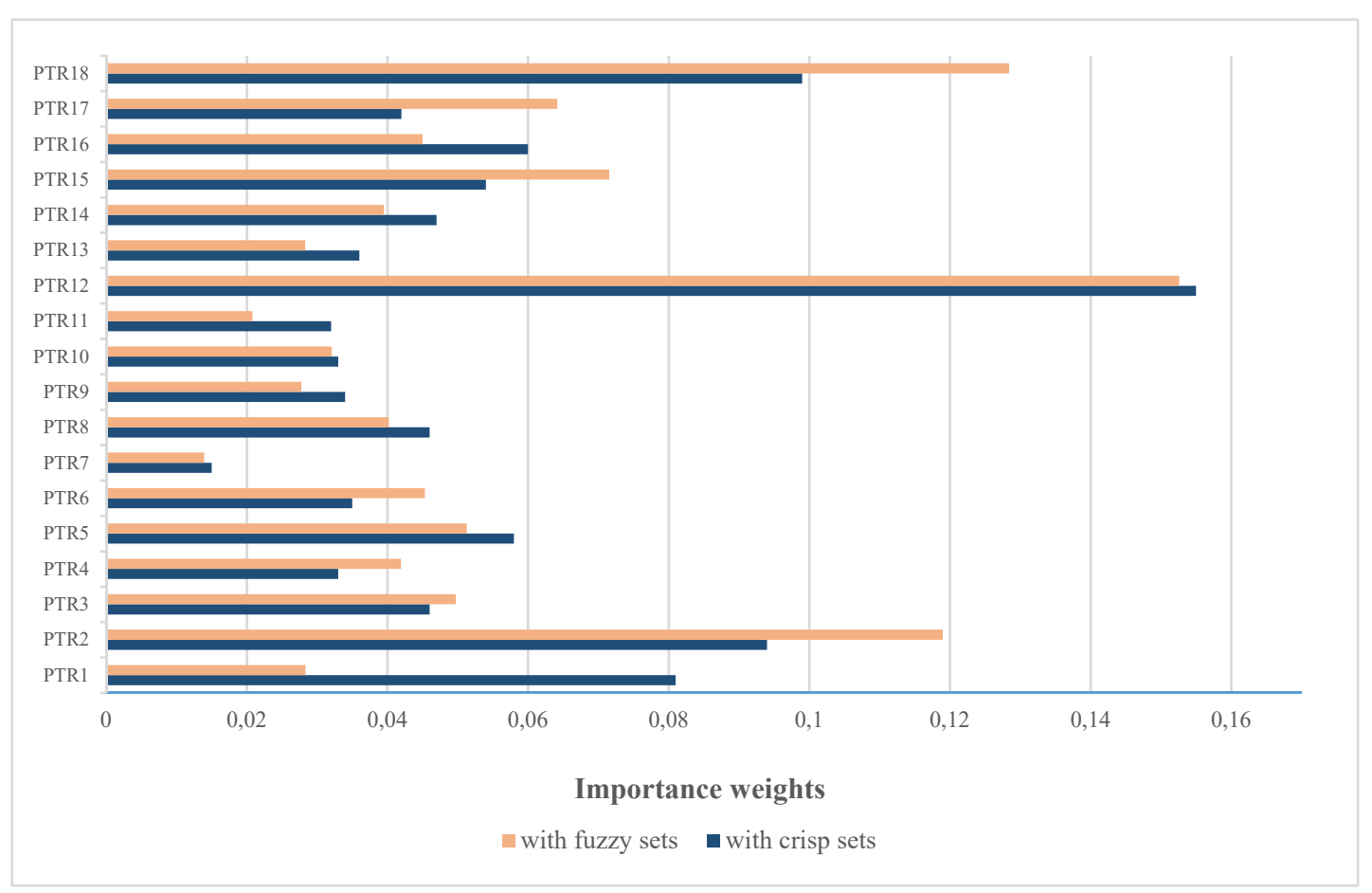

Fig. 8. Comparison of overall weighted priorities of PTRs 
We believe that simultaneously considering different goals has brought new directions to the product design process.

Figure 7, depicts the effect of different customer segments on the weighted overall ratings of PTRs. According to the findings, if the CSP wants to satisfy an academic institution as a customer, it should start paying attention to 'VM cost', 'CPU capacity' and 'security performance'. The results are effectively the same as for the first profile. However, the relative weights (\%21.41 versus \%15.26 for ' $V M$ cost') and their orders are different. Similarly, a commercial institution as a customer have different priorities and as a result, CSP should form a product focusing on these attributes; 'security performance', 'accountability' and 'VM cost' followed by the remaining attributes in a descending importance.

As the last analysis, we focus on the impact of fuzzy set theory usage on the results. In Figure 8, we compare the overall weights of the PTRs of a customer with Profile 1 in two different frameworks: The one, where only crisp numbers are used ${ }^{10}$, and the other where fuzzy triangular numbers are used. When we rank in terms of priorities, the first three PTRs are the same in both model; however there are quite a lot differences in the remaining 15 PTRs. The main benefit of incorporating fuzzy set theory into decision making process is to deal with the vagueness of the subjective judgments of various DMs. We believe the use of fuzzy set theory was necessary, since DMs were reluctant and/or unable to express their judgments using exact numbers.

\section{Conclusion}

In this paper, we proposed a product design decision support framework that incorporates different perspectives simultaneously. Undoubtedly, a successful cloud product should be able to satisfy its prospective customers in terms of quality merits it possesses. The identified customer needs for different customer profiles are the means for this purpose. However, this design also incorporates the price of the product (CN7 - ongoing cost), which is an essential quality attribute. Apart from customers, the same product should be able to satisfy its CSP. We think that two of the attributes perfectly capable of representing the CSPs' perspective are the cost and the sustainability. The cost view aims to obtain a costeffective product. Sustainability is a relatively new term that tries to achieve environmentally conscious and less resource consuming products and services. Sustainability is usually seen as a necessity for being a good corporate citizen. If the service provider is able to design a costoriented product, the result may a reduced price for the services provided. However, this perspective may ignore the customer expectations during the design phase. Hence, we tried to balance these different perspectives. We believe that if a CSP is able to consolidate these perspectives in its cloud offering when serving different customer segments by forming appropriate products, it will gain a competitive advantage in the cloud market. The relations between these goals/perspectives are presented using relative weights. These weights are used to prioritize the design process (Table 10). If the cloud service provider is inclined to satisfy customers, quality perspective should have a higher rating, which is the case in the demonstrative example. If the cloud service provider wants to build a product with low cost, then cost aim should have a higher rating. Hence, these ratings could be used to define a marketing strategy (build a product for low cost or build a product with differentiation in mind).

As a solution, we preferred QFD as the decision support tool, which examines customers' needs of different profiles and transform this subjective information into measurable product attributes. The end result is a tailored product for different customers. Herein, QFD provides a systematic procedure to associate various sources of input, both subjective (i.e. customer expectations and sustainability measures), and objective (i.e. product attributes and cost analysis results). Then, ANP is used for the pairwise comparisons required by QFD to deal with dependence issues, which are inevitable in such a complex decision problem. In all of the steps of the evaluation process, we applied fuzzy logic to deal with vague and imprecise information. We used Chang's fuzzy extent analysis to determine the final weights as crisp numbers. In this way, the subjective nature of the product design process has become a computationally systematic procedure.

As a future work, both the list of CNs and PTRs may be enhanced based on Cloud Service Measurement Index Consortium's measurement indexes 35 and ISO/IEC 25010:2011 standard. For the cost of the cloud product, we used comparative analysis to rate technical requirements; but a detailed analysis reflecting the dollar value of technical requirements could prove more useful. 
It is possible to apply the proposed framework to different services classes (XaaS) on condition of changing the evaluation criteria set.

\section{Acknowledgements}

This research has been financially supported by Galatasaray University Research Fund, with the project number [13.402.006].

\section{References}

1. X. Xu, From cloud computing to cloud manufacturing, Robotics and Computer-Integrated Manufacturing. 28(1) (2012) 175-186. doi: 10.1016/j.rcim.2011.07.002.

2. P. Mell, and T. Grance, The NIST Definition of Cloud Computing National Institute of Standards and Technology. (2011).

http://csrc.nist.gov/publications/nistpubs/800-145/SP800145.pdf.

3. C. N. Hoefer, and G. Karagiannis, Taxonomy of cloud computing services Journal of Internet Services and Applications. 2(2) (2011) 81-94. doi: 10.1007/s13174011-0027-x.

4. R. Buyya, C.S. Yeo, S. Venugopal, J. Broberg, and I. Brandic, Cloud Computing and Emerging IT Platforms: Vision, Hype, and Reality for Delivering Computing as the 5th Utility, Future Generation Computer Systems. 25(6) (2009) 599-616. doi: 10.1016/j.future.2008.12.001.

5. Gartner, Inc., Forecast: Public Cloud Services, Worldwide, 2014-2020, 2Q16 Update. (2016). https://www.gartner.com/doc/3357445/forecast-publiccloud-services-worldwide.

6. L. Leong, G. Petri, B. Gill, and M. Dorosh, Magic Quadrant for Cloud Infrastructure as a Service, Worldwide, Gartner Inc, (2016).

7. Amazon Continues to Gain Share in Cloud Infrastructure Services Market, Forbes August 17, (2016.)

8. A. Toffler, Future Shock, Bantam, Reissue edition, (1984).

9. G. Işıklar Alptekin, and S.E. Alptekin, A Knowledge Based Selection Framework for Cloud Services, in Proc. 2014 World Congress in Computer Science Computer Engineering and Applied Computing, (USA, Las Vegas, 2014), pp. 26-31.

10. G. Işıklar Alptekin, and S.E. Alptekin, Design of Customer-Oriented Products, in Proc. The World Congress on Engineering and Computer Science 2014 (WCECS 2014), (USA, San Francisco, 2014), pp. 724-729.

11. S. Akınlı Koçak, A. Miranskyy, G. Işıklar Alptekin, A.B. Bener, E. Cialini, The impact of improving software functionality on environmental sustainability, Information and Communication Technologies. (2013) 95-100.

12. T.L. Saaty, Decision Making with Dependence and Feedback: The Analytic Network Process (RWS Publications, Pittsburgh, 1996).

13. T.L. Saaty, The Analytic Hierarchy Process (McGrawHill, New York, 1980).
14. R.W. Saaty, Decision making in complex environments (Pittsburgh: Creative Decisions Foundation, 2003).

15. J.A. Carnevalli, and P.C. Miguel, Review, Analysis and Classification of the Literature on QFD - Types of Research, Difficulties and Benefits, International Journal Production Economics. 114(2) (2008) 737-754. doi: 10.1016/j.ijpe.2008.03.006.

16. N.M. Radwan, M.B. Senousy and A. El Din M. Riad, Approaches for Managing Uncertainty in Learning Management Systems, Egyptian Computer Science Journal 40 (2) (2016) 1-10.

17. L. Qu, Y. Wang, and M.A. Orgun, Cloud Service Selection Based on the Aggregation of User Feedback and Quantitative Performance Assessment, in Proc. the IEEE International Conference on Services Computing, (USA, Santa Clara, 2013) pp. 152-159. doi: 10.1109/SCC.2013.92.

18. M. Godse, and S. Mulik, An Approach for Selecting Software as a-Service (SaaS) Product, in Proc. the IEEE International Conference on Cloud Computing, (India, Bangalore, 2009) pp. 155-158. doi: 10.1109/CLOUD.2009.74.

19. C. Lo, D. Chen, C. Tsai, and K. Chao, Service Selection Based on Fuzzy TOPSIS Method, in Proc. the IEEE 24th International Conference on Advanced Information Networking and Applications Workshops, (Australia, Perth, 2010) pp. 367-372. doi: 10.1109/WAINA.2010.117.

20. M. Menzel, and R. Ranjan, CloudGenius: Decision Support for Web Server Cloud Migration, in Proc. the 21st International Conference on World Wide Web, (France, Lyon, 2012) pp. 979-988. doi: 10.1145/2187836.2187967.

21. S.K. Garg, S. Versteeg, and R. Buyya, A Framework for Ranking of Cloud Computing Services, Future Generation Computer Systems. 29(4) (2013) 1012-1023. doi: 10.1016/j.future.2012.06.006.

22. D. Ergu, and Y. Peng, A framework for SaaS Software Packages Evaluation and Selection with Virtual Team and BOCR of Analytic Network Process, The Journal of Supercomputing. 67(1) (2014) 219-238. doi: 10.1007/s11227-013-0995-7.

23. H.T. Liu, and C.H. Wang, An advanced quality function deployment model using fuzzy analytic network process, Applied Mathematical Modelling 34(11) (2010), 33333351.

24. S. Zaim, M. Sevkli, H. Camgöz-Akdağ, O.F. Demirel, A.Y. Yayla and D. Delen, Use of ANP weighted crisp and fuzzy QFD for product development, Expert Systems with Applications 41(9) (2014), 4464-4474.

25. T. Ertay, G. Büyükozkan, C. Kahraman, D. Ruan, Quality function deployment implementation based on analytic network process with linguistic data: An application in automative industry, Journal of Intelligent \& Fuzzy Systems 16(3) (2005) 221-232.

26. L.K. Chan, and M.L. Wu, A Systematic Approach to Quality Function Deployment with a Full Illustrative 
Example, Omega. 33(2) (2005) 119-139. doi: 10.1016/j.omega.2004.03.010.

27. E.W.L. Cheng, H. Li, and L. Yu, The Analytic Network Process (ANP) Approach to Location Selection: A Shopping Mall Illustration, Construction Innovation. 5(2) (2004) 83-97. doi: 10.1108/14714170510815195.

28. L.A. Zadeh, Fuzzy sets, Information and Control, 8 (1965) 338-353. doi:10.1016/S0019-9958(65)90241-X.

29. D.Y. Chang, Applications of the extent analysis method on fuzzy AHP, European Journal of Operational Research. 95 (1996) 649-655. doi:10.1016/0377-2217(95)00300-2.

30. C. Kahraman, T. Ertay, and G. Buyukozkan, Fuzzy optimization model for QFD planning process using analytic network approach, European Journal of Operational Research. $171 \quad$ (2006) 390-411. http://dx.doi.org/10.1016/j.ejor.2004.09.016.

31. E.E. Karsak, S. Sozer, and S.E. Alptekin, Product planning in quality function deployment using a combined analytic network process and goal programming approach, Computers \& Industrial Engineering. 44(1) (2003) 171190. doi: 10.1016/S0360-8352(02)00191-2.

32. T.L. Saaty, and L.G. Vargas, Diagnosis with Dependent Symptoms: Bayes Theorem and the Analytic Hierarchy Process, Operations Research. 46(4) (1998) 491-502. doi: 10.1287/opre.46.4.491.

33. T.L. Saaty, and M. Takizawa, Dependence and Independence: From Linear Hierarchies to Nonlinear Networks, European Journal of Operational Research. 26(2) (1986) 229-237. doi: 10.1016/0377-2217(86)901840 .

34. G.A. Miller, The Magical Number Seven, Plus or Minus Two: Some Limits on our Capacity for Processing Information, Psychological Review. 63(2) (1956) 81-97.

35. Cloudcommons, Service Measurement Index Version 1.0, Cloudcommons. Accessed September 4. (2011) http://www.cloudcommons.com/documents/10508/186d5 f13-f40e-47ad-b9a6-4f246cf7e34f. 